\title{
Energy loss in gas-surface dynamics: electron-hole pair and phonon excitation upon adsorbate relaxation
}

\author{
D. Novko ${ }^{\mathrm{a}, *}$, M. Blanco-Rey ${ }^{\mathrm{b}, \mathrm{a}}$, J.I. Juaristi ${ }^{\mathrm{b}, \mathrm{c}, \mathrm{a}}$, M. Alducin $^{\mathrm{c}, \mathrm{a}}$ \\ ${ }^{a}$ Donostia International Physics Center (DIPC), Paseo Manuel de Lardizabal 4, 20018 Donostia-San Sebastián, \\ Spain \\ ${ }^{b}$ Departamento de Física de Materiales, Facultad de Químicas UPV/EHU, Apartado 1072, 20080 Donostia-San \\ Sebastián, Spain \\ ${ }^{c}$ Centro de Física de Materiales CFM/MPC (CSIC-UPV/EHU), Paseo Manuel de Lardizabal 5, 20018 \\ Donostia-San Sebastián, Spain
}

\begin{abstract}
We study the effect of electron and phonon degrees of freedom on the relaxation dynamics of adsorption processes in gas-surface systems by using ab initio molecular dynamics that incorporates an electronic friction force (AIMDEF). As representative cases we have chosen three systems with different adsorption energies and adsorbate-to-surface atom mass ratios: $\mathrm{H}$ on $\mathrm{Pd}(100), \mathrm{N}$ on $\operatorname{Ag}(111)$, and $\mathrm{N}_{2}$ on $\mathrm{Fe}(110)$. We show, through inspection of the total energies and trajectories of the hot adsorbates on the surface, that electron-hole (e-h) pair excitations dominate relaxation of the light gas species, while the phonon channel is dominant for the heavy species. In the latter case e-h pairs become more important at the final thermalization stages.
\end{abstract}

Keywords: Energy Loss, Electronic Friction, Metal Surfaces, Hot Atoms and Molecules, Density Functional Theory

PACS: 82.65.+r, 34.35.+a, 34.50.Bw, 68.43.-h

\section{Introduction}

During the adsorption of gas atoms or molecules on surfaces, the involved gas species have to dissipate their excess kinetic energy, which is sometimes increased by the surface attraction, into the two following energy loss channels: electron-hole (e-h) pair excitations and surface vibrations (phonons). During this relaxation process, the adsorbed hot species propagate on the surface, and their energy loss is dictated by the interplay between the e-h pair and phonon dissipation channels. Evi-

\footnotetext{
${ }^{*}$ Corresponding author

Email address: dino_novko001@ehu.eus (D. Novko)

Preprint submitted to Elsevier
}

dence of e-h pair excitations in the adsorption of thermal gas particles on metal surfaces is manifested experimentally in the socalled chemicurrents, which are electrical currents generated upon adsorption of atomic and molecular species on metal surfaces [1-3]. In other experiments the excited electrons are observed when the highly vibrational excited molecules collide with the metal surface $[4,5]$. The efficiency of the energy loss channels in dissipating the excess kinetic energy of the incoming particles will determine, for instance, whether the hot species can recombine with another adsorbate or whether thermalization occurs before recombination.

In a recent work, it was demonstrated to what extent e-h pairs and phonons dominate

February 3, 2016 
the relaxation process in gas-surface systems with a wide range of adsorption energies, $E_{\text {ads }}$, and adsorbate-to-surface atom mass ratios, $\gamma=$ $m_{\mathrm{A}} / m_{\mathrm{S}}$ [6]. The chosen systems were hot $\mathrm{H}$ atoms on $\operatorname{Pd}(100)$ that originate from the dissociative adsorption of $\mathrm{H}_{2}(\gamma=0.0094$ and $\left.E_{\text {ads }}=0.5 \mathrm{eV}\right)$, atomic $\mathrm{N}$ on $\mathrm{Ag}(111)(\gamma=0.13$ and $\left.E_{\text {ads }}=2.2 \mathrm{eV}\right)$, and $\mathrm{N}_{2}$ molecules on $\mathrm{Fe}(110)\left(\gamma=0.5\right.$ and $\left.E_{\text {ads }}=0.2-0.5 \mathrm{eV}\right)$. For $\mathrm{H}$ on $\mathrm{Pd}(100)$ it was shown that e-h pair excitations are the dominant energy loss channel [7] and that they dissipate energy at a five times faster rate than the phonons channel [8]. For heavier adsorbates, like $\mathrm{N}$ and $\mathrm{N}_{2}$, it was shown that the phonon energy loss channel dominates, while the effect of the smaller but continuous dissipation into e-h pair excitations becomes apparent at long time scales.

In this paper, we revisit the above mentioned systems to analyse the problem from the point of view of the influence of e-h pairs and phonons on the adsorbates total energies and their trajectories. In particular, we are interested in learning on the mean traveled distance of the hot species on the surface. In principle, the in-plane distance between the initial and final positions of the adsorbate on the surface can be experimentally measured with scanning tunneling microscopy (STM) $[9,10]$. To obtain the above mentioned quantities we have employed the same state of the art methods used in Refs. $[6,7]$ to perform the classical molecular dynamics (MD) at ab initio level.

This paper is organized as follows. In Sec. 2 we introduce the methodology and the results and discussions follow in Sec. 3. In Sec. 4 we give the conclusions.

\section{Methods}

In $a b$ initio molecular dynamics (AIMD) simulations, the adiabatic forces between the atoms in the system are calculated at each integration step of the classical dynamics equations using the Hellmann-Feynman theorem and density functional theory (DFT). This enables the description of the surface atom movements (phonons) in each of the simulation step. Additionally, in the recently implemented AIMD with electronic friction (AIMDEF) [7], the nonadiabatic effects that come from the energy exchange between nuclear and electronic degrees of freedom are accounted through an electronic friction force included into the classical equations of motion [14]. To describe this electronic friction force at the position $\mathbf{r}_{\mathrm{A}}$ of the projectile, $-\eta\left(\mathbf{r}_{\mathrm{A}}\right) \dot{\mathbf{r}}_{\mathrm{A}}$, we use the local density friction approximation (LDFA) [15]. In this approach, the friction coefficient $\eta\left(\mathbf{r}_{\mathrm{A}}\right)$ at each time step of the simulation is uniquely a function of the bare surface electronic density at the position $\mathbf{r}_{\mathrm{A}}, \rho_{\text {surf }}\left(\mathbf{r}_{\mathrm{A}}\right)$. More precisely, $\eta\left(\mathbf{r}_{\mathrm{A}}\right)$ is calculated for an atom traveling through a homogeneous free electron gas [16-19] with electronic density equal to $\rho_{\text {surf }}\left(\mathbf{r}_{\mathrm{A}}\right)$. In case of a molecular projectile, the independent atom approximation is additionally performed, in which $\eta\left(\mathbf{r}_{\mathrm{A}}\right)$ is calculated for each atom of the molecule independently. The LDFA is widely used to treat the effect of e-h pair excitations in many of the elementary processes that involve atoms and molecules at metal surfaces, e.g., adsorption and scattering events $[6,7,15,20-26]$, recombination and desorption processes [2729], as well as for quantifying the vibrational lifetime of adsorbates [30-32].

In simulations where the surface atoms are allowed to move, the surface electronic density will correspondingly vary with time. Thus, application of the LDFA requires in those cases the knowledge at any instant $t$ of the bare surface electronic density, $\rho_{\text {surf }}\left(\mathbf{r}_{\mathrm{A}}, t\right)$. The latter complicates the implementation of the LDFA in usual AIMDEF, because it is the electronic density of the whole system, i.e., gas species and surface atoms, and not the bare surface electronic density, what is self-consistently calculated at each integration step. This problem was tackled in Ref. [6], where the Hirshfeld partitioning scheme [33] was used to construct the bare surface electronic density on the fly. In 
Table 1: DFT computational details for $\mathrm{H} / \mathrm{Pd}(100), \mathrm{N} / \mathrm{Ag}(111)$ and $\mathrm{N}_{2} / \mathrm{Fe}(110)$ : functionals, energy cut-offs for the plane wave basis set $\left(E_{\text {cut-off }}\right)$, cell sizes, numbers of surface layers, Monkhorst-Pack $(M P)$ meshes for the Brillouin zone, and spin polarization.

\begin{tabular}{cccc}
\hline & $\mathrm{H} / \mathrm{Pd}(100)$ & $\mathrm{N} / \mathrm{Ag}(111)$ & $\mathrm{N}_{2} / \mathrm{Fe}(110)$ \\
\hline Functional & $\mathrm{PW} 91[11]$ & $\mathrm{RPBE}[12]$ & $\mathrm{RPBE}$ \\
$E_{\text {cut-off }}$ & $350 \mathrm{eV}$ & $400 \mathrm{eV}$ & $400 \mathrm{eV}$ \\
cell size & $2 \times 2$ & $2 \times 2$ & $3 \times 3$ \\
\# layers & 5 & 5 & 4 \\
MP mesh [13] & $6 \times 6 \times 1$ & $5 \times 5 \times 1$ & $3 \times 3 \times 1$ \\
spin-polarized & $\mathrm{NO}$ & $\mathrm{YES}$ & $\mathrm{YES}$ \\
\hline
\end{tabular}

this embedding scheme the contribution of the adsorbate to the electronic density is removed from the self-consistent (SCF) electron density of the whole system, $\rho^{\mathrm{SCF}}\left(\mathbf{r}_{\mathrm{A}}\right)$, at each time step of the simulation. The surface electron density then reads

$$
\begin{aligned}
\rho_{\text {surf }}\left(\mathbf{r}_{\mathrm{A}}\right) & =\rho^{\mathrm{SCF}}\left(\mathbf{r}_{\mathrm{A}}\right)\left[1-\sum_{n}^{N_{\mathrm{A}}} w_{n}\left(\mathbf{r}_{\mathrm{A}}\right)\right], \\
w_{n}\left(\mathbf{r}_{\mathrm{A}}\right) & =\frac{\rho_{n}^{\text {atom }}\left(\mathbf{r}_{\mathrm{A}}\right)}{\sum_{m}^{N} \rho_{m}^{\text {atom }}\left(\mathbf{r}_{\mathrm{A}}\right)},
\end{aligned}
$$

where $N$ and $N_{\mathrm{A}}$ are the total numbers of atoms in the system and in the adsorbate, respectively, and $\rho_{n}^{\text {atom }}$ is the electron density of the isolated $n$-th atom. The weighting factor $w_{n}\left(\mathbf{r}_{\mathrm{A}}\right)$ specifies the relative share at $\mathbf{r}_{\mathrm{A}}$ of the $n$-th atom in the electron density of the whole system. Thus, the factor in square brackets of Eq. (1) defines the weight corresponding to the system without the contribution of the adsorbate. Recently, the Hirshfeld partitioning scheme was used to calculate the vibrational lifetimes of molecular adsorbates within the LDFA framework [32].

In the present work, all the simulations were performed using the DFT-based Vienna Ab initio Simulation Package VASP [34], which uses a plane wave basis set. The electroncore interaction was described by projector augmented wave potentials [35]. In each of the studied systems we use a different general- ized gradient approximation (GGA) for the exchange and correlation functional, as detailed in Table 1. The functionals were chosen to facilitate the comparison of our AIMDEF calculations with previous works [7, 36-38]. Further computational details can be found in Table 1 . The classical equations of motion were integrated using the Beeman's predictor-corrector method for velocities [39]. The integration time steps are $0.1 \mathrm{fs}$ for $\mathrm{H} / \mathrm{Pd}(100), 0.5$ fs for $\mathrm{N} / \mathrm{Ag}(111)$, and $0.7 \mathrm{fs}$ for $\mathrm{N}_{2} / \mathrm{Fe}(110)$. These time steps were selected so that the drift in the total energy is negligible when compared to the corresponding energy loss due to e-h pair excitations.

In order to disentangle the relevance of each energy dissipation channel, we perform AIMDEF simulations in the frozen surface $(\mathrm{FS}+\mathrm{EF})$ and the non-frozen surface (NFS+EF) approaches. In the latter, the atoms in the two topmost layers were allowed to move during the simulation run. Non-frozen surface AIMD simulations without electronic friction (NFS) were carried out as well.

The simulations of hot $\mathrm{H}$ atom dynamics on the $\operatorname{Pd}(100)$ surface were performed with the initial coordinates and velocities corresponding to the already dissociated $\mathrm{H}_{2}$ molecule on $\operatorname{Pd}(100)$, but keeping the $\mathrm{H}$ atoms independent from each other. These initial conditions were taken from adiabatic frozen surface MD simulations done on a six-dimensional potential energy surface (PES) (see Ref. [7, 40]), where 
the $\mathrm{H}_{2}$ molecule approaches the surface with $E_{i}\left(\mathrm{H}_{2}\right)=0.5 \mathrm{eV}$ at normal incidence. For each method, 50 trajectories were simulated. Spin unpolarized calculations are justified here because, after dissociation, the $\mathrm{H}$ atoms propagate close to the surface and thus the spin is quenched.

The adsorption dynamics of $\mathrm{N}$ on $\mathrm{Ag}(111)$ was studied for normal incidence and initial kinetic energy $E_{i}=0.1 \mathrm{eV}$. These incidence conditions were shown to provide a high adsorption probability of around 0.9 [36]. A total of 20 trajectories, which start at a distance $z_{i}=4 \AA$ above the surface, were simulated with randomly chosen initial lateral positions $\left(x_{i}, y_{i}\right)$.

In the case of non-dissociative adsorption of a $\mathrm{N}_{2}$ molecule on $\mathrm{Fe}(110)$, the sticking probability at normal incidence is maximised by an initial energy $E_{i}=0.75 \mathrm{eV}[37,38]$. A total of 80 trajectories starting from $z_{i}=4 \AA$ above the surface were run with random initial $\left(x_{i}, y_{i}\right)$ values and orientations.

\section{Results and discussion}

\subsection{Total energy of the gas species}

In Figs. 1(a), 2(a) and 3(a)-(c) we show the total energy of the adsorbates (A), $E_{\text {tot }}(\mathrm{A})$, as a function of integration time and averaged over the total number of trajectories. The total energy here is the sum of the kinetic and potential energies of the adsorbate, $E_{\text {tot }}(\mathrm{A})=$ $E_{\text {kin }}(\mathrm{A})+E_{\text {pot }}(\mathrm{A})$. For all the cases the zero of potential energy is defined as the potential energy of the adsorbate when it is located in vacuum. For the $\mathrm{H}$ atom we also substrate one half of the $\mathrm{H}_{2}$ binding energy in the gas phase to that reference potential, which is calculated considering the spin polarization of the $\mathrm{H}$ atom placed in the vacuum, in order to have the $\mathrm{H}$ atom in the $\mathrm{H}_{2}$ molecule located far from $\operatorname{Pd}(100)$ as the potential energy reference.

The kinetic energy term $E_{\text {kin }}(\mathrm{A})$ is obtained on the fly during the simulation, but the potential energy term $E_{\text {pot }}(\mathrm{A})$ needs to be calculated from a posteriori analysis of the available trajectories. The reason is that the total potential energy obtained from the simulations varies not only due to the adsorbates movement, but also due to the lattice distortion. Thus, $E_{\text {pot }}(\mathrm{A})$ is obtained as follows. First, we remove the adsorbate from the system for a few time instants $\left\{t_{n}\right\}$, leaving only the distorted surface. For each $\left\{t_{n}\right\}$, we calculate the average over the available trajectories of the substrate (S) potential energy increase with respect to the equilibrium bare surface configuration, $E_{\text {pot }}\left(\mathrm{S} ; t_{n}\right)$. Then, we linearly interpolate them to get the $E_{\text {pot }}(\mathrm{S} ; t)$ values at each time step of the simulation ${ }^{1}$. Since we know the potential energy of the whole system $E_{\text {pot }}(\mathrm{A}+\mathrm{S} ; t)$ from the AIMDEF simulations, we can evaluate the potential energy of the adsorbate at each time step as,

$$
E_{\text {pot }}(\mathrm{A} ; t)=E_{\text {pot }}(\mathrm{A}+\mathrm{S} ; t)-E_{\text {pot }}(\mathrm{S} ; t) .
$$

By looking at the total energy of the $\mathrm{H}$ atom moving on $\operatorname{Pd}(100)$ we observe a considerably larger decay rate when electronic friction is included in the calculation (FS+EF and NFS+EF) than when it is absent (NFS). The same dominant role of e-h pair excitations in this case was seen in the kinetic energy of the $\mathrm{H}$ atom $[6,7]$. In this system $\mathrm{H}$ adsorbs at the hollow sites, which are sketched by a red cross in the inset of Fig. 1(c). The horizontal black dashed line in Fig. 1(a) shows the potential energy of the $\mathrm{H}$ atom relaxed at the bridge site [yellow circle of Fig. 1(c) inset] calculated with Eq. (2), $E_{\text {pot }}^{\text {bri }}(H)$. The latter represents the energy threshold for a $\mathrm{H}$ atom to jump from one hollow site to the adjacent one. In our simulations the $\mathrm{H}$ atom closely reaches this point at around $0.5 \mathrm{ps}$ after dissociation, but only in the NFS+EF case. The red dotted line marks

\footnotetext{
${ }^{1}$ The $\left\{t_{n}\right\}$ points used for interpolation of $E_{\mathrm{pot}}\left(\mathrm{S} ; t_{n}\right)$ are $\{0,0.08,0.16,0.24,0.32,0.4,0.48,0.5\},\{0,0.5,1.0$, $1.5,2.0,2.5,3.0,3.5\}$, and $\{0,0.07,0.175,0.35,0.525$, $0.7,1.4,2.1,2.8,3.5,4.2\}$ ps for $\mathrm{H} / \mathrm{Pd}(100), \mathrm{N} / \mathrm{Ag}(111)$, and $\mathrm{N}_{2} / \mathrm{Fe}(110)$, respectively.
} 
the $\mathrm{H}$ atom potential energy relaxed at the hollow site $E_{\mathrm{pot}}^{\mathrm{hol}}(\mathrm{H})$, i.e. the thermalised situation, which lies close to the $\mathrm{H}$ propagation threshold for this system.

For the case of $\mathrm{N} / \mathrm{Ag}(111)$ the relaxation dynamics is governed by the energy transfer to the lattice, as it can be inferred from the faster decay of the NFS and NFS+EF curves with respect to the FS+EF one. However, the e-h pair excitations still play an important role. While the difference between NFS and NFS+EF simulations was not so noticeable in the kinetic energy decay rate in Ref. [6], here it is clear that $E_{\text {tot }}(\mathrm{N})$ goes through a faster decay when the electronic energy loss is included. Generally this means that e-h pair excitations are not only affecting the kinetic energy, but also the potential energy because they modify the trajectory. There are two hollow adsorption sites for the $\mathrm{N}$ atom, namely $h c p$ and $f c c$ represented by a red triangle and a green cross, respectively, in the inset of Fig. 2(c). Similar to the $\mathrm{H} / \mathrm{Pd}(100)$ case, the energy threshold for the $\mathrm{N}$ atom to jump from one hollow site to the neighbouring one over the bridge site, $E_{\mathrm{pot}}^{\mathrm{bri}}(\mathrm{N})$, is almost reached for the NFS+EF at the end of the simulation run [see the black dashed line in Fig. 2(a)]. Nevertheless, the relaxed N potential energies at the $f c c$ and $h c p$ sites [see the green dotted and red dashed-dotted lines in Fig. 2(a), respectively] indicate that the $\mathrm{N}$ atom still needs to lose a few hundred meV to be fully thermalised at either hollow site. As we will show below, electronic excitations play an important role in the accommodation of the adsorbate in the adsorption well [36].

$\mathrm{N}_{2}$ adsorption on the $\mathrm{Fe}(110)$ surface is characterized by the three distinct adsorption configurations at top, hollow and bridge sites that are sketched in Fig. 3 [6, 37, 38]. Since the adsorption properties are quite different in each case, we find it meaningful to separate the analysis of this system according to the final accommodation site. The results in Figs. 3(a)(c) show that, independently of the adsorp- tion site, $\mathrm{N}_{2} / \mathrm{Fe}(110)$ is also a system where the phonon energy loss channel dominates over the e-h pair excitations. Actually, the role of phonons in this system is crucial because there is no adsorption in the FS+EF case. It can be seen that the total energy of $\mathrm{N}_{2}$ reaches the smallest value for the hollow site, while for the other two sites it remains slightly larger. This is mostly due to the different adsorption energies of $\mathrm{N}_{2}$. The largest value is found at the hollow site $\left(E_{\mathrm{ads}}^{\text {hol }}=0.49 \mathrm{eV}\right)$, while smaller values are found for the top $\left(E_{\mathrm{ads}}^{\mathrm{top}}=0.31 \mathrm{eV}\right)$ and bridge $\left(E_{\mathrm{ads}}^{\mathrm{bri}}=0.22 \mathrm{eV}\right)$ sites. Interestingly, at the end of the simulation, the total energy of those molecules that end up on the top site [Fig. 3(a)] is still larger than for the ones that end up on the bridge site [Fig. 3(c)]. This result, which clearly contrasts with the values of $E_{\text {ads }}$, shows that thermalization at the top well is much slower than at the bridge one. This happens because both energy loss channels depend strongly on the adsorption configuration. At the top site, the molecule lies further from the surface, where the electron density is lower and thus the e-h pair excitations are less efficient. The comparison of the NFS curves in Figs. 3(a) and (c) shows that the phonon excitation channel is also less efficient at the top site. However, the relationship between adsorption configuration and lattice excitations is less straightforward to understand here than in the electronic channel case. Finally, from the potential energies given by Eq. (2) for the molecule relaxed at each adsorption site, which are indicated by dotted lines in Figs. 3(a) and (c), we see that the molecules of the bridge site are the closest to thermalisation at the end of the simulation time.

\subsection{Lateral displacements on the surface}

Here we analyse the role of e-h pair and phonon energy loss channels on the propagation of adsorbates on the surface for the three case studies. We calculate the averaged lateral distance, $d$, traveled by the adsorbate from its initial in-plane coordinates $\left(x_{i}, y_{i}\right)$ up to the 
(a)

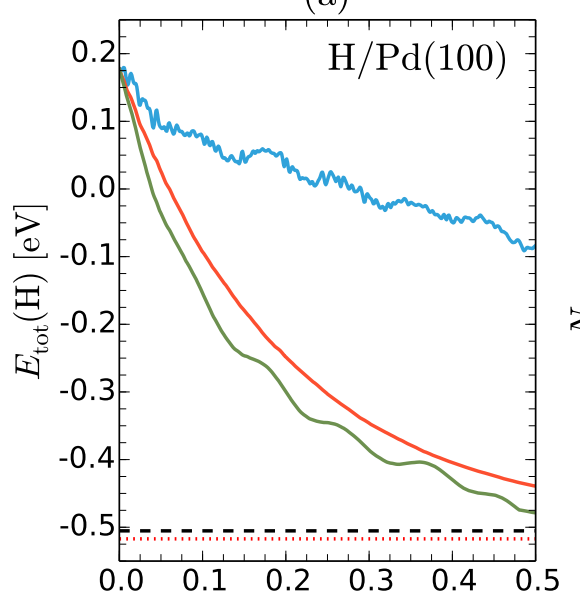

(b)

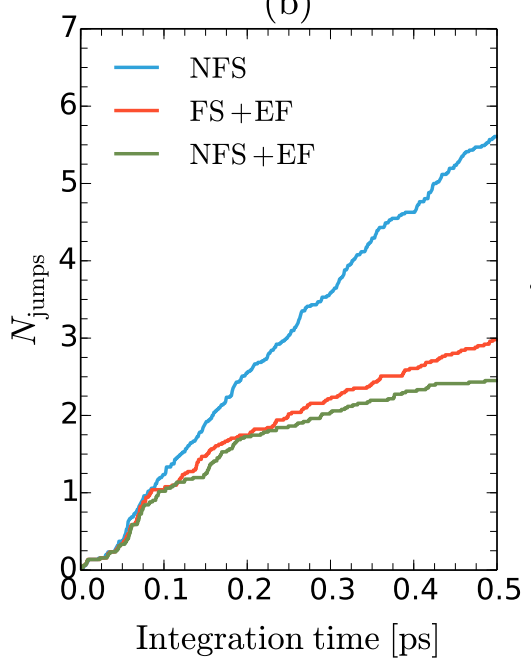

(c)

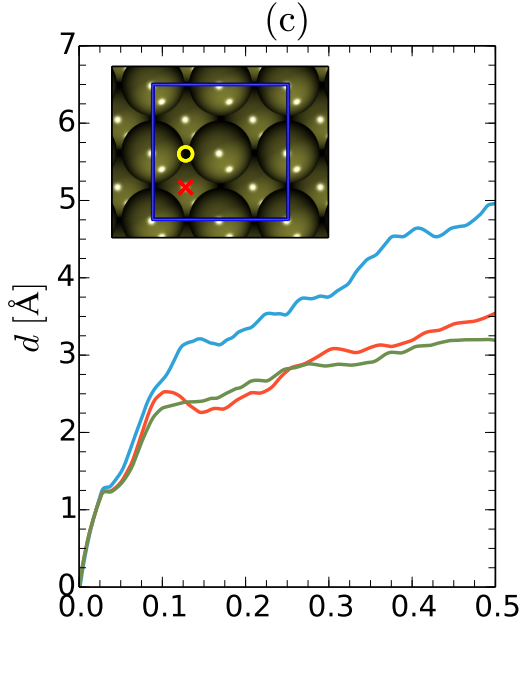

Figure 1: (a) Average total energy of the $\mathrm{H}$ atom on the $\mathrm{Pd}(100)$ surface, $E_{\mathrm{tot}}(\mathrm{H})$, (b) number of jumps between the hollow sites, $N_{\text {jump }}$, and (c) lateral displacement from the initial position, $d$, as a function of integration time. Blue, red and green curves represent NFS, FS+EF and NFS+EF calculations, respectively. The horizontal black dashed line indicates the energy needed for the $\mathrm{H}$ atom to jump between two adjacent hollow sites of the $\mathrm{Pd}(100)$ surface, this is, the potential energy of the $\mathrm{H}$ atom (see Eq. (2)) relaxed at the bridge site, $E_{\mathrm{pot}}^{\mathrm{bri}}(\mathrm{H})$. The horizontal red dotted line indicates the potential energy at the adsorption hollow site $E_{\mathrm{pot}}^{\text {hol }}(\mathrm{H})$. Inset: $2 \times 2$ unit cell of the $\mathrm{Pd}(100)$ surface (blue box) with a red cross representing the hollow site and a yellow circle at the bridge site.

end of the trajectories. In the case of the hot $\mathrm{H}$ and $\mathrm{N}$ atoms, for which the quite intricate adsorption paths are characterized by larger lateral displacements than for $\mathrm{N}_{2}$ adsorption, we also calculate the averaged number of jumps between adjacent hollow sites, $N_{\text {jumps }}$. These two values along with the knowledge of $E_{\text {tot }}(\mathrm{A})$ allow a complete characterization of the hot species lateral movement on the surface, which is relevant for the reactivity properties.

Figs. 1(b) and 1(c) show the time evolution of $N_{\text {jumps }}$ and $d$, respectively, for $\mathrm{H} / \mathrm{Pd}(100)$. We see that both quantities rapidly increase in the first $0.1 \mathrm{ps}$. It is during this time interval that the total energy of $\mathrm{H}$ is almost reduced by a half of the total energy lost at the end of simulation. The importance of e-h pair excitations in the relaxation process is also clearly visible in the trajectories. When the electronic energy loss channel is active, we observe a significant reduction in $N_{\text {jumps }}$ and $d$ by almost a factor of 2 and 1.5, respectively, at the final simulation time. Since the H atom in the NFS+EF simu- lations is close to the propagation threshold set by $E_{\text {pot }}^{\text {bri }}(\mathrm{H})$ [see Fig. 1(a)], we can consider that the values of $d$ and $N_{\text {jumps }}$ are basically close to saturation. With this information, we can establish that the final averaged number of jumps between neighbouring hollow sites will be between 2 and 3, while the lateral distance from the starting position will be around $3 \AA[7]$.

The dominant role of the phononic energy loss channel in the $\mathrm{N} / \operatorname{Ag}(111)$ system, which was discussed in Sec. 3.1, is also apparent in Figs. 2(b) and 2(c), as a large difference in $N_{\text {jumps }}$ and $d$ between the cases where the phonon energy loss channel is active (NFS and $\mathrm{NFS}+\mathrm{EF})$ and where it is not (FS+EF). We also observe that e-h pair excitations play a significant role in the difference between $N_{\text {jumps }}$ obtained with the NFS and with the NFS+EF simulations, which amounts to $\Delta N_{\text {jumps }} \approx 5$ in the final stage of the simulation. This difference is less noticeable for $d$ because the values in both cases are very similar and almost saturated after around 2 ps. However, the averaged 
(a)

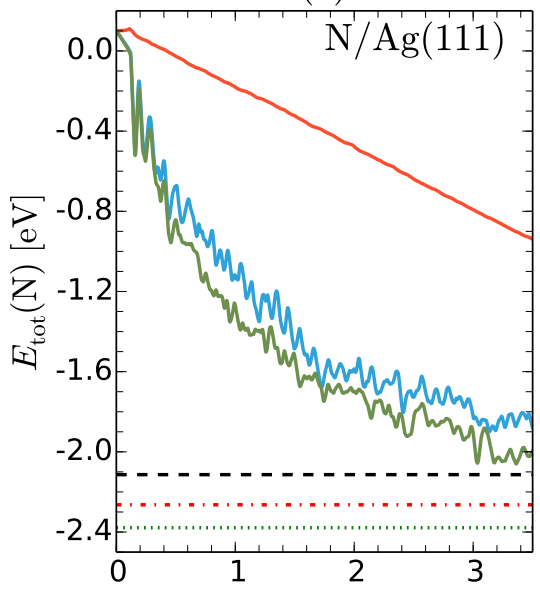

(b)

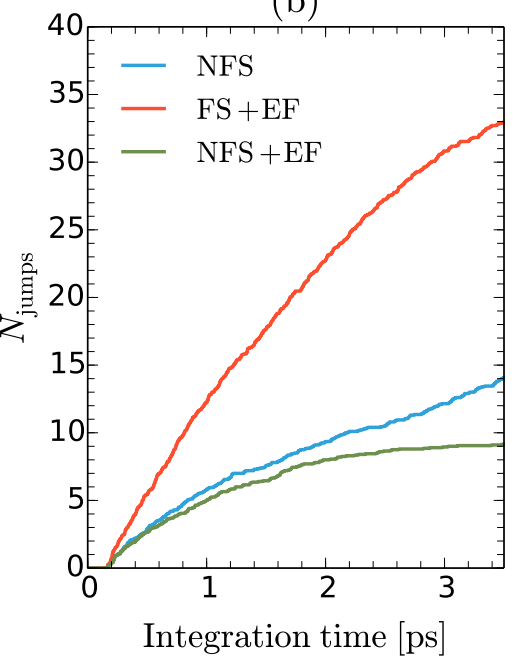

(c)

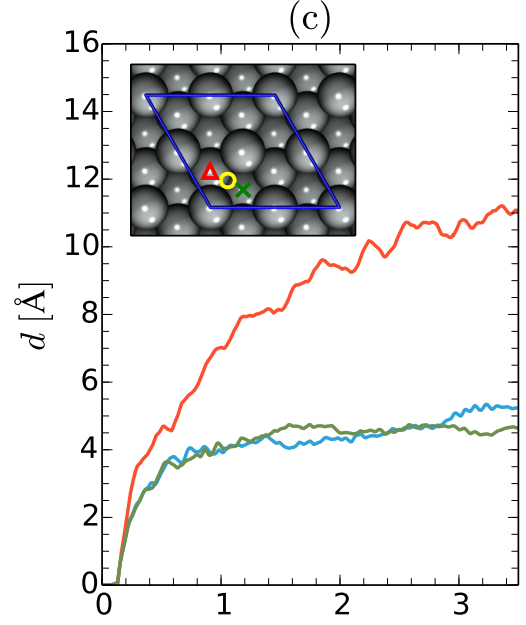

Figure 2: (a) Average total energy of the $\mathrm{N}$ atom on the $\operatorname{Ag}(111)$ surface, $E_{\mathrm{tot}}(\mathrm{N}),(\mathrm{b})$ number of jumps between the hollow sites, $N_{\text {jump }}$, and (c) lateral displacement from the initial position, $d$, as a function of integration time. Line colors description as in Fig. 1. The horizontal black dashed line indicates the energy needed for a $\mathrm{N}$ atom to jump between two adjacent hollow sites of the $\mathrm{Ag}(111)$ surface, this is, the potential energy of $\mathrm{N}$ (see Eq. (2)) relaxed at the bridge site, $E_{\mathrm{pot}}^{\mathrm{bri}}(\mathrm{N})$. The same quantity at the $f c c$ and $h c p$ adsorption sites is indicated by a green dotted and a red dashed-dotted line, respectively. Inset: $2 \times 2$ unit cell of the $\operatorname{Ag}(111)$ surface (blue box) with a green cross and a red triangle at the hollow $f c c$ and $h c p$ sites, respectively, and a yellow circle at the bridge site.

traveled-path length $L_{\text {ads }}$ at the end of the integration time is $4.5 \AA$ longer in the NFS than in the NFS+EF simulations (not shown in the figure). First of all, the differences obtained in $N_{\text {jumps }}$ and $L_{\text {ads }}$ between both calculations show the importance of e-h pair excitations in the accommodation process of the $\mathrm{N}$ atom on the adsorption well $[6,36]$. And secondly, the saturation of $d$ reached after 2 ps in comparison to the still increasing behaviour of $N_{\text {jumps }}$ (and also of $L_{\text {ads }}$ ) tells us that $\mathrm{N}$ is hopping while conserving on average the distance to the initial position. The fact that the $E_{\mathrm{pot}}^{\mathrm{bri}}(\mathrm{N})$ threshold for $\mathrm{N}$ hopping is almost reached explains the saturation of $N_{\text {jumps }}$ and $d$ by the end of the simulation run in the NFS+EF case, as discussed above. By comparing $N_{\text {jumps }}$ and $d$ for this case with the $\mathrm{H} / \mathrm{Pd}(100)$ case, it is clear that the $\mathrm{N}$ atom propagates longer distances than the $\mathrm{H}$ atom, which is primarily due to the difference in adsorption energies between these two cases.

Figures 3(d)-(f) show the time dependence of the statistically averaged lateral distance of the $\mathrm{N}_{2}$ center of mass from its initial position $\left(x_{i}, y_{i}\right), d_{\text {c.m. }}$, for the three adsorption sites. We have checked that the $\mathrm{N}_{2}$ molecule barely makes jumps between adsorption sites (not shown) and that it arrives quite fast at the maximum lateral distance. The latter happens at around $0.5 \mathrm{ps}$ for all the adsorption sites and it is due to the repulsive character of the PES for the approaching molecule [37, 38], which strongly reduces the total energy in the first collision with the surface (see Figs 3(a)-(c)). Afterwards, the molecules undergo small lateral displacements mostly due to movement at the adsorption site. For the molecules adsorbed at the top site this can be observed through oscillations of $d_{\text {c.m. }}$ for $t \gtrsim 0.5 \mathrm{ps,} \mathrm{which} \mathrm{mostly}$ come from the combination of frustrated translational modes and precession of the molecular center of mass on top of the Fe atom. We see that molecules ending up at the top sites reach the largest $d_{\text {c.m. }}$, while those ending up at hollow or bridge sites the smallest. This is due 

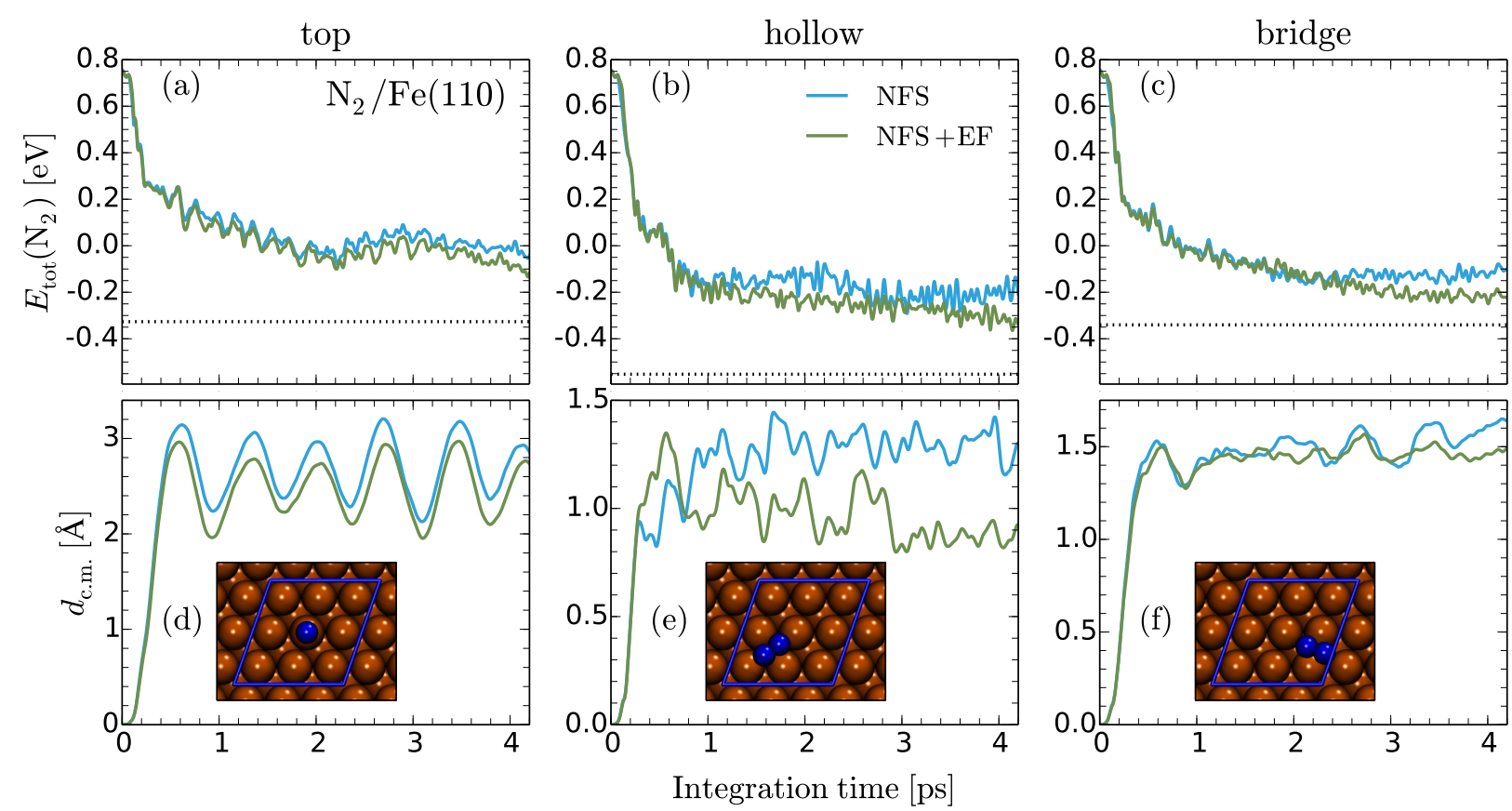

Figure 3: Average total energy of $\mathrm{N}_{2}$ on $\mathrm{Fe}(110), E_{\text {tot }}\left(\mathrm{N}_{2}\right)$, and lateral displacement of the molecules center of mass from the initial position, $d_{\text {c.m. }}$, as a function of the integration time for molecules that end up on (a) top, (b) hollow, and (c) bridge sites. Line colors description as in Fig. 1. The horizontal dotted lines indicate the potential energy of the molecule at each adsorption site, which is calculated using Eq. (2). Inset: $3 \times 3$ unit cell of the Fe(110) surface (blue box) with a $\mathrm{N}_{2}$ molecule adsorbed on each site.

to the PES profile: at the top site $\mathrm{N}_{2}$ lies upright and bound to one Fe atom, while it lies parallel and tightly bound to the surface at the other two sites due to the bonds that it forms with more than one Fe atom. As for the difference between the NFS and NFS+EF calculations, we observe that the excitation of e-h pairs causes in general a decrease in $d_{\text {c.m. }}$. The largest reduction of around $0.5 \AA$ occurs for the hollow site, i.e., the configuration that, having the largest $E_{\text {ads }}$ and being close to the surface, where the electron density is high, better fulfills the conditions for an efficient excitation of e-h pairs.

\section{Conclusion}

We have performed AIMDEF simulations to analyse the thermalization process in three representative cases of gas-surface interaction: $\mathrm{H} / \mathrm{Pd}(100), \mathrm{N} / \mathrm{Ag}(111)$ and $\mathrm{N}_{2} / \mathrm{Fe}(110)$. In the first system a dominant role is played by e$\mathrm{h}$ pair excitations and in the other two systems by phonon excitations, although e-h pairs are still important for the accommodation of the gas species in the final adsorption site.

We calculated the total energy of the adsorbate and examined the hot species trajectories on the surfaces. We found that, for the $\mathrm{H} / \mathrm{Pd}(100)$ system, the lateral displacement of the $\mathrm{H}$ atoms starts to saturate at $\sim 0.5 \mathrm{ps}$ after dissociation, when the total energy becomes close to the energy threshold for the $\mathrm{H}$ atom to hop from one adsorption site to another one. During this time period the adsorbate does not propagate much, reaching the final position at $\sim 3 \AA$ from its initial position and making around 2 jumps between adjacent adsorption sites. In the $\mathrm{N} / \mathrm{Ag}(111)$ system we also observed how the lateral displacement reaches its maximum value when the mentioned threshold 
energy is reached. In this system the adsorbates undergo larger lateral displacements than in the $\mathrm{H} / \mathrm{Pd}(100)$ system due to its larger adsorption energy. In contrast, the $\mathrm{N}_{2}$ molecule arrives early at the final adsorption site and does not propagate much after this point. For this last studied case we have separated the analysis according to the final adsorption site. The molecules that end up adsorbed upright at the top site travel longer distances on the surface than the ones which end up lying parallel at hollow and bridge sites. The reason is that the molecule is bound to various Fe atoms in the latter two cases, what strongly reduces its mobility. Our results suggest that in the systems with light gas species a considerable increase of dissipation rate of adsorbates total energy and a decrease of lateral displacements exist when the e-h pair energy loss channel is active. For heavier gas species the excitation of e-h pairs is important for the final accommodation in the adsorption well.

\section{Acknowledgments}

This work has been supported by the Basque Departamento de Educación, Universidades e Investigación, the University of the Basque Country UPV/EHU (Grant No. IT-756-13) and the Spanish Ministerio de Economía y Competitividad (Grant No. FIS2013-48286C2-2-P). The authors thankfully acknowledge the computer resources, technical expertise and assistance provided by the Red Española de Supercomputación and by the DIPC computing center.

[1] H. Nienhaus, H. Bergh, B. Gergen, A. Majumdar, W. Weinberg, E. McFarland, Direct detection of electronhole pairs generated by chemical reactions on metal surfaces, Surf. Sci. 445 (2000) 335. doi:10.1016/S0039-6028(99)01078-X.

[2] B. Gergen, H. Nienhaus, W. H. Weinberg, E. W. McFarland, Chemically induced electronic excitations at metal surfaces, Science 294 (2001) 2521. doi:10.1126/science.1066134.

[3] H. Nienhaus, Electronic excitations by chemical reactions on metal surfaces, Surf. Sci. Rep. 45 (2002) 1. doi:10.1016/S0167-5729(01)00019-X.
[4] Y. Huang, C. T. Rettner, D. J. Auerbach, A. M. Wodtke, Vibrational promotion of electron transfer, Science 290 (2000) 111. doi:10.1126/science.290.5489.111.

[5] J. D. White, J. Chen, D. Matsiev, D. J. Auerbach, A. M. Wodtke, Conversion of large-amplitude vibration to electron excitation at a metal surface, Nature 433 (2005) 503. doi:10.1038/nature03213.

[6] D. Novko, M. Blanco-Rey, J. I. Juaristi, M. Alducin, Ab initio molecular dynamics with simultaneous electron and phonon excitations: Application to the relaxation of hot atoms and molecules on metal surfaces, Phys. Rev. B 92 (2015) 201411. doi:10.1103/PhysRevB.92.201411.

[7] M. Blanco-Rey, J. I. Juaristi, R. Díez Muiño, H. F. Busnengo, G. J. Kroes, M. Alducin, Electronic friction dominates hydrogen hot-atom relaxation on Pd(100), Phys. Rev. Lett. 112 (2014) 103203. doi:10.1103/PhysRevLett.112.103203.

[8] A. Gross, Ab Initio molecular dynamics study of hot atom dynamics after dissociative adsorption of $\mathrm{H}_{2}$ on $\mathrm{Pd}(100)$, Phys. Rev. Lett. 103 (2009) 246101. doi:10.1103/PhysRevLett.103.246101.

[9] B. C. Stipe, M. A. Rezaei, W. Ho, S. Gao, M. Persson, B. I. Lundqvist, Single-molecule dissociation by tunneling electrons, Phys. Rev. Lett. 78 (1997) 4410. doi:10.1103/PhysRevLett.78.4410.

[10] J. R. Hahn, W. Ho, Orbital specific chemistry: Controlling the pathway in single-molecule dissociation, J. Chem. Phys. 122 (2005) 244704. doi:10.1063/1.1940007.

[11] J. P. Perdew, J. A. Chevary, S. H. Vosko, K. A. Jackson, M. R. Pederson, D. J. Singh, C. Fiolhais, Atoms, molecules, solids, and surfaces: Applications of the generalized gradient approximation for exchange and correlation, Phys. Rev. B 46 (1992) 6671. doi:10.1103/PhysRevB.46.6671.

[12] B. Hammer, L. B. Hansen, J. K. Nørskov, Improved adsorption energetics within densityfunctional theory using revised perdew-burkeernzerhof functionals, Phys. Rev. B 59 (1999) 7413. doi:10.1103/PhysRevB.59.7413.

[13] H. J. Monkhorst, J. D. Pack, Special points for brillouin-zone integrations, Phys. Rev. B 13 (1976) 5188. doi:10.1103/PhysRevB.13.5188.

[14] M. Head-Gordon, J. C. Tully, Molecular dynamics with electronic frictions, J. Chem. Phys. 103 (1995) 10137. doi:10.1063/1.469915.

[15] J. I. Juaristi, M. Alducin, R. Díez Muiño, H. F. Busnengo, A. Salin, Role of electronhole pair excitations in the dissociative adsorption of diatomic molecules on metal surfaces, Phys. Rev. Lett. 100 (2008) 116102. doi:10.1103/PhysRevLett.100.116102.

[16] P. M. Echenique, R. M. Nieminen, R. H. Ritchie, Density functional calculation of stopping power of 
an electron gas for slow ions, Solid State Commun. 37 (1981) 779. doi:10.1016/0038-1098(81)91173-X.

[17] P. M. Echenique, R. M. Nieminen, J. C. Ashley, R. H. Ritchie, Nonlinear stopping power of an electron gas for slow ions, Phys. Rev. A 33 (1986) 897. doi:10.1103/PhysRevA.33.897.

[18] A. Salin, A. Arnau, P. M. Echenique, E. Zaremba, Dynamic nonlinear screening of slow ions in an electron gas, Phys. Rev. B 59 (1999) 2537. doi:10.1103/PhysRevB.59.2537.

[19] H. Winter, J. I. Juaristi, I. Nagy, A. Arnau, P. M. Echenique, Energy loss of slow ions in a nonuniform electron gas, Phys. Rev. B 67 (2003) 245401. doi:10.1103/PhysRevB.67.245401.

[20] I. Goikoetxea, J. I. Juaristi, M. Alducin, R. Díez Muiño, Dissipative effects in the dynamics of $\mathrm{n} 2$ on tungsten surfaces, J. Phys.: Condens. Matter 21 (26) (2009) 264007.

[21] L. Martin-Gondre, M. Alducin, G. A. Bocan, R. Díez Muiño, J. I. Juaristi, Competition between electron and phonon excitations in the scattering of nitrogen atoms and molecules off tungsten and silver metal surfaces, Phys. Rev. Lett. 108 (2012) 096101. doi:10.1103/PhysRevLett.108.096101.

[22] A. S. Muzas, J. I. Juaristi, M. Alducin, R. Díez Muiño, G. J. Kroes, C. Díaz, Vibrational deexcitation and rotational excitation of $\mathrm{h} 2$ and $\mathrm{d} 2$ scattered from $\mathrm{cu}(111)$ : Adiabatic versus nonadiabatic dynamics, J. Chem. Phys. 137 (6) (2012) 064707. doi:http://dx.doi.org/10.1063/1.4742907.

[23] L. Martin-Gondre, G. A. Bocan, M. Blanco-Rey, M. Alducin, J. I. Juaristi, R. Díez Muiño, Scattering of nitrogen atoms off $\operatorname{ag}(111)$ surfaces: A theoretical study, J. Phys. Chem. C 117 (2013) 9779-9790. doi:10.1021/jp312667w.

[24] M. Pavanello, D. J. Auerbach, A. M. Wodtke, M. Blanco-Rey, M. Alducin, G. J. Kroes, Adiabatic energy loss in hyperthermal $\mathrm{h}$ atom collisions with cu and au: A basis for testing the importance of nonadiabatic energy loss, J. Phys. Chem. Lett. 4 (2013) 3735.

[25] O. Bünermann, H. Jiang, Y. Dorenkamp, A. Kandratsenka, S. Janke, D. J. Auerbach, A. M. Wodtke, Electron-hole pair excitation determines the mechanism of hydrogen atom adsorption, Science 350 (6266) (2015) 1346-1349. doi:10.1126/science.aad4972.

[26] B. Jiang, M. Alducin, H. Guo, Electron-hole pair effects in polyatomic dissociative chemisorption: Water on ni(111), J. Phys. Chem. Lett. 7 (2016) 327-331. doi:10.1021/acs.jpclett.5b02737.

[27] O. Galparsoro, R. Pétuya, J. I. Juaristi, C. Crespos, M. Alducin, P. Larregaray, Energy dissipation to tungsten surfaces upon eley-rideal recombination of $\mathrm{n} 2$ and $\mathrm{h} 2$, J. Phys. Chem. C 119 (27) (2015) 15434. doi:http://dx.doi.org/10.1021/acs.jpcc.5b04286.

[28] G. Füchsel, T. Klamroth, S. Monturet, P. Saalfrank, Dissipative dynamics within the electronic friction approach: the femtosecond laser desorption of h2/d2 from ru(0001), Phys. Chem. Chem. Phys. 13 (2011) 8659-8670. doi:10.1039/C0CP02086A.

[29] I. Lonćaric, M. Alducin, P. Saalfrank, J. I. Juaristi, Femtosecond-laser-driven molecular dynamics on surfaces: Photodesorption of molecular oxygen from ag(110), Phys. Rev. B 93 (2016) 014301. doi:10.1103/PhysRevB.93.014301.

[30] J. C. Tremblay, S. Monturet, P. Saalfrank, Electronic damping of anharmonic adsorbate vibrations at metallic surfaces, Phys. Rev. B 81 (2010) 125408. doi:10.1103/PhysRevB.81.125408.

[31] P. Saalfrank, J. I. Juaristi, M. Alducin, M. BlancoRey, R. Díez Muiño, Vibrational lifetimes of hydrogen on lead films: An ab initio molecular dynamics with electronic friction (aimdef) study, J. Chem. Phys. 141 (23) (2014) 234702. doi:10.1063/1.4903309.

[32] S. P. Rittmeyer, J. Meyer, J. I. Juaristi, K. Reuter, Electronic friction-based vibrational lifetimes of molecular adsorbates: Beyond the independentatom approximation, Phys. Rev. Lett. 115 (2015) 046102. doi:10.1103/PhysRevLett.115.046102.

[33] F. L. Hirshfeld, Bonded-atom fragments for describing molecular charge densities, Theoret. Chim. Acta 44 (1977) 129. doi:10.1007/BF00549096.

[34] G. Kresse, J. Hafner, Ab initio molecular dynamics for liquid metals, Phys. Rev. B 47 (1993) 558. doi:10.1103/PhysRevB.47.558.

[35] P. E. Blöchl, Projector augmented-wave method, Phys. Rev. B 50 (1994) 17953. doi:10.1103/PhysRevB.50.17953.

[36] L. Martin-Gondre, G. A. Bocan, M. Alducin, J. I. Juaristi, R. Díez Muiño, Energy dissipation channels in the adsorption of $\mathrm{N}$ on $\mathrm{Ag}(111)$, Comp. Theo. Chem. 990 (2012) 126. doi:10.1016/j.comptc.2012.03.009.

[37] I. Goikoetxea, M. Alducin, R. Díez Muiño, J. I. Juaristi, Dissociative and non-dissociative adsorption dynamics of $\mathrm{N}_{2}$ on $\mathrm{Fe}(110)$, Phys. Chem. Chem. Phys 14 (2012) 7471. doi:10.1039/C2CP40229G.

[38] I. Goikoetxea, J. I. Juaristi, R. Díez Muiño, M. Alducin, Surface strain improves molecular adsorption but hampers dissociation for $\mathrm{N}_{2}$ on the Fe/W(110) surface, Phys. Rev. Lett. 113 (2014) 066103. doi:10.1103/PhysRevLett.113.066103.

[39] D. Beeman, Some multistep methods for use in molecular dynamics calculations, J. Comput. Phys. 20 (1976) 130. doi:10.1016/00219991(76)90059-0. 
[40] A. Lozano, A. Gross, H. F. Busnengo, Adsorption dynamics of $\mathrm{H}_{2}$ on $\mathrm{Pd}(100)$ from first principles, Phys. Chem. Chem. Phys. 11 (2009) 5814. doi:10.1039/B905432B. 